\title{
Microtomography versus Optical Microscopy in the Examination of Interior Features in Ice
}

\author{
R. W. Obbard,* and I. Baker*
}

* Thayer School of Engineering at Dartmouth College, 8000 Cummings Hall, Hanover, NH 03755

Visible microinclusions in glacial ice cores, including dust and volcanic debris, tiny cracks and voids, can be examined in detail and identified nondestructively using microtomography. This technique is especially useful for features, such as pores, where in situ analysis is required. Several types of voids are found in deep polar cores: bubbles, clathrates, and flat plate-like voids [1]. Bubbles formed by pore closeoff in the top of the glacier, shrink with depth and finally disappear. Starting at around a thousand feet, entrained gas exists only in solution or in clathrate cages [2-3]. Bubbles and clathrates in glacial ice cores are of interest to climatologists because they hold information about the climate at the time of original deposition [1,4]. When ice cores are retrieved, the reduced pressure permits gas to come out of solution and gather in flat plate-like voids [5-6]. These tell us something about the conditions in the glacier itself, and the thermal history of the core.

Because of the transparent nature of ice, optical microscopy may be used to image inclusions and catalog pore shape and size [7]. Bubble and dust number density and mean size can be determined through optical microscopy and laser light scattering and pore volume and sample density can be calculated through physical tests [8]. With the advent of micro computer tomography, however, has come the ability to better measure microinclusions and study their distribution in three dimensions. We compare and contrast optical and chromatographic images of a core collected from the Vostok site on the East Antarctic ice sheet to illustrate the methods'comparative benefits and drawbacks.

This core contains small $(50 \mu \mathrm{m})$ hexagonal voids that lie on the basal planes of the grains in which they are found and that produce reflections in the ice (Fig. 1). These can be clearly distinguished from bubbles in an optical microscope (Fig. 2). Microtomography is used to distinguish bubbles from hexagonal voids and to illustrate their distribution in three dimensional space (Fig. 3), and reveals that what appeared as a crack in the sample (Fig. 1) is actually a plane of microbubbles [9].

References

[1] A. J. Gow and T. Williamson, J. Geophys. Res. 80 (1975) 5101.

[2] S. L. Miller, Science. 165 (1969) 489.

[3] F. Pauer et al., J. Glaciol. 45(149) (1999) 22.

[4] M. K. Spencer et al., J. Glaciol. 52(178) (2006) 358.

[5] A. J. Gow, J. Geophys. Res. 76(11) (1971) 2533.

[6] J. Muguruma et al., Phil. Mag. 13 (1966) 625.

[7] F. Pauer et al., Polarforschung. 66(3) (1996) 31.

[8] M. Ram and G. Koenig, J. Geophys. Res. 102 (1997) 26641.

[9] This research was supported by the U.S. National Science Foundation (NSF) Grant OPP

0440523. The views and conclusions contained herein are those of the authors and should not be interpreted as necessarily representing official policies, either expressed or implied, of the NSF or the U.S. Government. 

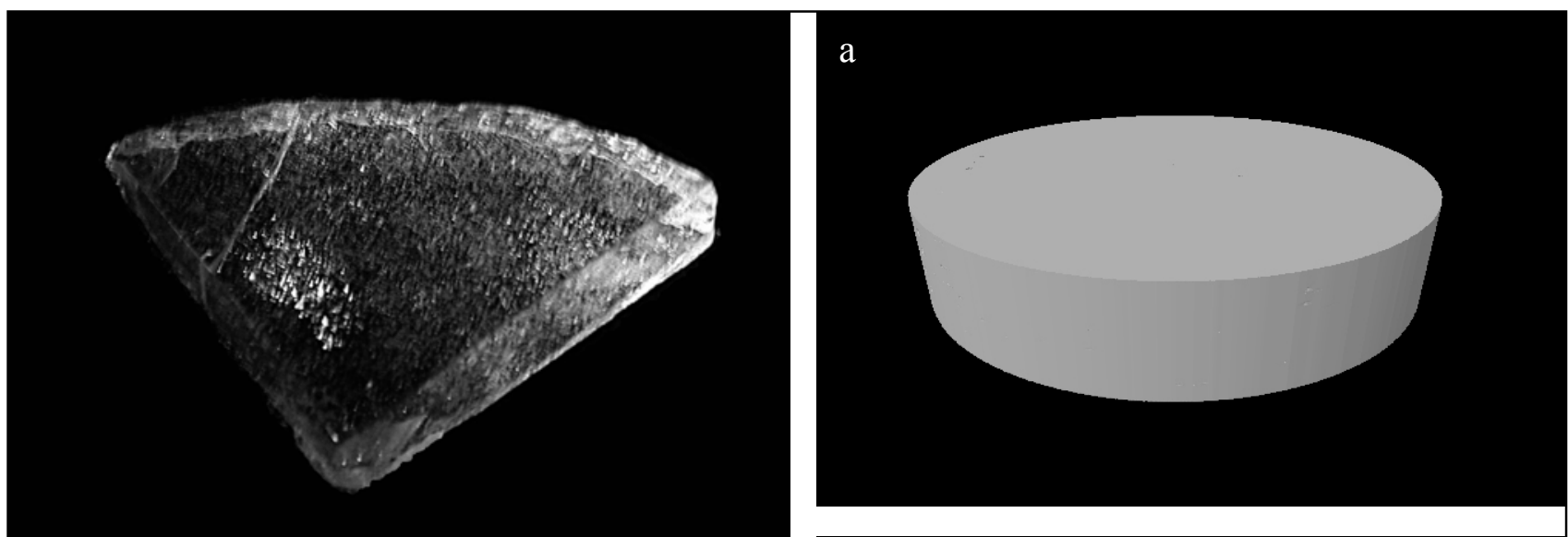

Figure 1. Section of polycrystalline Vostok (Antarctica) ice core from $3399 \mathrm{~m}$. Reflective areas inside are groups of hexagonal plate shaped voids lying on the basal plane of a crystal. The line across the left corner that appears to be a crack is shown in more detail in Fig 3b, c, and d.
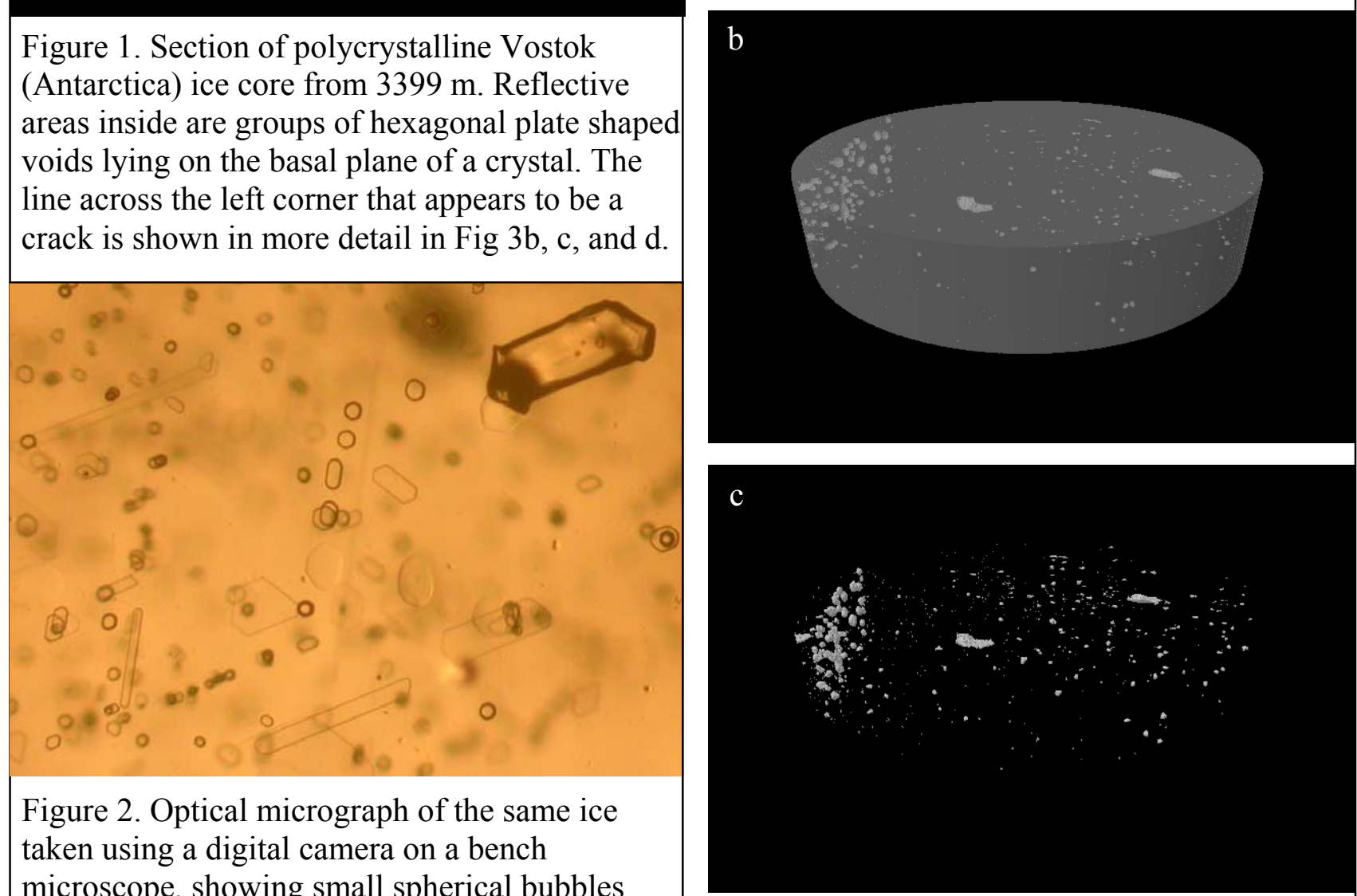

Figure 2. Optical micrograph of the same ice taken using a digital camera on a bench microscope, showing small spherical bubbles and one large and many small flat polygonal voids. Magnification $65 \mathrm{X}$.

Figure 3. (right) Four variants of a single microtomographic scan of Vostok 3399 m core ice, showing scattered small bubbles, two larger voids, and a plane of bubbles. The $6 \mathrm{~mm}$ disk is a region of interest chosen from a larger specimen. In $\mathrm{c}$ and $\mathrm{d}$, the image is reversed to show only the pore space.

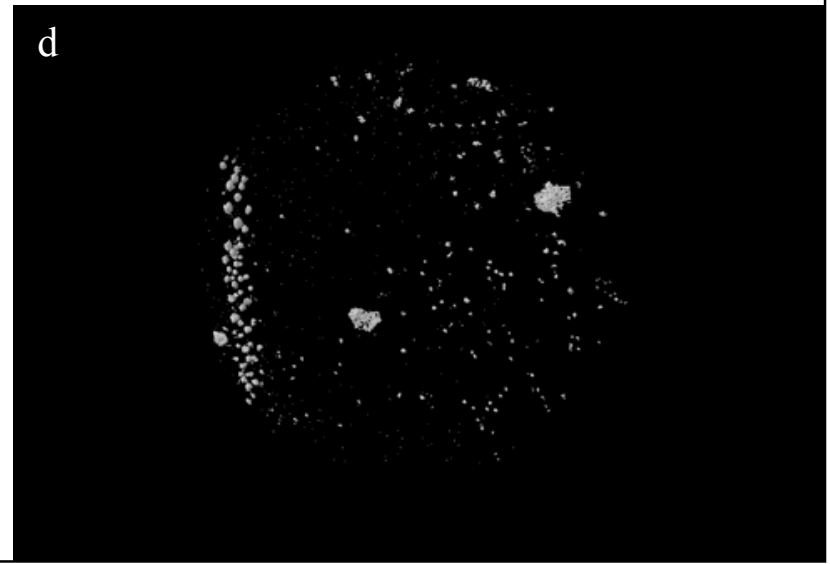

